\title{
Self-reported Sleep Impairment in Functional Dyspepsia and Irritable Bowel Syndrome
}

\author{
Seung Young Kim, ${ }^{1}$ Rok Seon Choung, ${ }^{2}$ Seung Ku Lee, Jung Wan Choe, ${ }^{3}$ Sung Woo Jung, ${ }^{3}$ Jong Jin Hyun, ${ }^{1}$ Ja Seol Koo, \\ Sang Woo Lee, ${ }^{1}$ and Chol Shin ${ }^{3 *}$ \\ ${ }^{1}$ Division of Gastoenterology and Hepatology, Department of Internal Medicine, Korea University Ansan Hospital, Ansan, Korea; ${ }^{2}$ Division of \\ Gastroenterology and Hepatology, Mayo Clinic, Rochester, Minnesota, USA; and ${ }^{3}$ Institute of Human Genomic Study, Korea University Ansan \\ Hospital, Ansan, Korea
}

\section{Background/Aims \\ Sleep impairment is a common complaint among patients with irritable bowel syndrome (IBS) and functional dyspepsia (FD). This study aimed to evaluate the prevalence of sleep impairment in FD or IBS patients, and to determine whether IBS-FD overlap induced more sleep disturbance than FD or IBS alone.}

\section{Methods}

A population-based cohort in South Korea including 2251 subjects was asked about gastrointestinal symptoms including IBS and dyspepsia-related symptoms. In addition, sleep disturbance was measured using the Pittsburgh Sleep Quality Index and Epworth Sleepiness Scale questionnaires. One-way ANOVA and logistic regression were used to assess differences among the 4 groups (healthy subjects, IBS alone, FD alone, and IBS-FD overlap).

\section{Results}

Of 2251 subjects who were surveyed by questionnaire, 2031 responded (92.5\% response rate) and were analyzed. The prevalence of IBS, FD, and IBS-FD overlap was 8.0\% (95\% confidence interval [Cl], 6.8-9.2\%), 4.8\% (95\% Cl, 3.9-5.8\%), and 1.8\% (95\% Cl, 1.2$2.4 \%)$, respectively. FD alone, but not IBS alone, was significantly associated with a poorer sleep quality index $(\mathrm{OR}, 2.68 ; 95 \% \mathrm{Cl}, 1.43-$ 5.01) and more daytime sleepiness (OR, 2.21; $95 \% \mathrm{Cl}, 1.14-4.30)$, compared to healthy subjects. IBS-FD overlap had the greatest likelihood of a poorer sleep quality index (OR, 3.88; $95 \% \mathrm{Cl}, 1.83-8.19)$, daytime sleepiness $(\mathrm{OR}, 2.47 ; 95 \% \mathrm{Cl}, 1.01-5.67)$, and insomnia (OR, 2.84; $95 \% \mathrm{Cl}, 1.39-5.82)$, compared to healthy subjects.

\section{Conclusion}

A correlation between functional gastrointestinal disorders and sleep disturbance was demonstrated, which was significantly pronounced in the context of IBS-FD overlap.

(J Neurogastroenterol Motil 2018;24:280-288)

\section{Key Words}

Functional gastrointestinal disorder; Irritable bowel syndrome; Sleep

Received: August 25, 2017 Revised: November 18, 2017 Accepted: December 12, 2017

(5) This is an Open Access article distributed under the terms of the Creative Commons Attribution Non-Commercial License (http://creativecommons. org/licenses/by-nc/4.0) which permits unrestricted non-commercial use, distribution, and reproduction in any medium, provided the original work is properly cited.

${ }^{*}$ Correspondence: Chol Shin, MD, PhD

Division of Pulmonary, Sleep and Critical Care Medicine, Department of Internal Medicine, Korea University Ansan Hospital and Institute of Human Genome Study, 123 Jukgumro, Danwon-gu, Ansan, Gyeonggi-do 15355, Korea Tel: +82-31-412-5603, Fax: +82-31-412-5604, E-mail: chol-shin@hanmail.net

Seung Young Kim and Rok Seon Choung contributed equally to this work. 


\section{Introduction}

The incidence of sleep-related problems, such as sleep loss and chronic insomnia, is increasing in modern society. Sleep disorders are associated with increased morbidities, as evidenced by the increased need for general care and mental health treatment. ${ }^{1,2}$ Sleep disturbances are common in patients with gastrointestinal symptoms and a few reports have shown the association between sleep impairment and gastrointestinal disease, such as peptic ulcer, ${ }^{3}$ gastroesophageal reflux disease, ${ }^{4}$ and functional gastrointestinal disorders (FGIDs), including irritable bowel syndrome (IBS) and functional dyspepsia (FD). ${ }^{5}$ Several studies that investigated the prevalence of sleep disturbance in FD or IBS found that it was higher in FD patients than in controls, ${ }^{6}$ and that these disorders were associated with gastrointestinal symptom severity; ${ }^{7}$ however, most of these studies were limited by small sample sizes.

On the other hand, FD and IBS are reported to overlap frequently, ${ }^{8}$ ince various gastrointestinal symptoms appear at the same time in a patient. In a cross-sectional study including 2680 subjects in a health check-up population, Kaji et al $^{9}$ reported that the prevalence of FD, IBS, and IBS-FD overlap was $10.0 \%, 14.2 \%$, and $3.4 \%$, respectively. However, few reports have investigated concurrent sleep disturbance and IBS-FD overlap.

Therefore, we aimed to evaluate the prevalence of sleep impairment in FD or IBS patients, and to determine whether IBS-FD overlap induces more sleep disturbance than FD or IBS alone.

\section{Materials and Methods}

\section{Study Subjects}

All study subjects were derived from the Ansan cohort of the Korean Genomic Epidemiology Study (KoGES), an ongoing population-based cohort study designed to establish a representative adult cohort in the city of Ansan, South Korea and to identify the epidemiologic characteristics and the frequency and determinants of chronic disease in Koreans. Detailed information on the KoGES study design and aims have been previously reported. ${ }^{10}$ From June 2001 to January 2003, a longitudinal cohort was formed, consisting of 5012 participants (2518 men and 2494 women aged 40-69 years) who participated in a comprehensive health examination and on-site interviews at Korea University Ansan Hospital. They were followed up biennially. Among 3021 subjects who participated in the 6th 2nd examination in 2012 and 7th 1st examination in 2013,
2251 subjects (1161 men and 1090 women) were asked about gastrointestinal symptoms according to the Rome III criteria. We excluded participants who did not complete questionnaires ( $\mathrm{n}=$ 169) or who had cancers $(\mathrm{n}=51)$. We included 2031 subjects (1070 men and 961 women) in this analysis. This study was approved by the Institutional Review Board of Korea University Ansan Hospital (ED0624).

\section{Definition}

FD and IBS were defined according to the Rome III criteria. ${ }^{11,12} \mathrm{FD}$, diagnosis was based on one or more of the following symptoms for the prior 3 months, with symptom onset at least 6 months earlier: bothersome postprandial fullness, early satiation, epigastric pain, or epigastric burning with no evidence of structural disease that could explain the symptoms. ${ }^{11}$ The IBS diagnosis was based on the presence of recurrent abdominal pain or discomfort associated with 2 or more of the following: improvement after defecation, onset associated with a change in frequency of stools, or onset associated with a change in stool form for the prior 3 months, with symptom onset at least 6 months prior to diagnosis. ${ }^{12}$ IBS-FD overlap was defined as the fulfillment of both IBS and FD criteria by the same patient.

\section{Questionnaire on Lifestyle and Anthropometric and Biochemical Data}

The questionnaire included questions about smoking status (never or current), alcohol consumption (never or current), income (monthly wage of $<200,200-400,>400 \times 10^{4}$ Korean won), physical activity (metabolic equivalents per hour daily), occupation (white collar or blue collar), education level $(<6,6-12,>12$ years), and marital status. Body mass index (BMI) was calculated as weight in kilograms divided by the square of height in meters. Blood pressure was measured by trained technicians using a mercury sphygmomanometer, with the subject in the supine position after at least a 10-minute rest period. Blood samples were taken in the morning after an overnight fast. We measured fasting glucose, serum triglycerides, total-cholesterol, and high-density lipoprotein cholesterol.

\section{Questionnaires on Depression and Quality of Life}

The Beck Depression Inventory (BDI), a 21-item self-reported inventory, is used to assess the severity of depressive symptoms. ${ }^{13}$ Participants were asked to indicate which statement best described the way they have been feeling for the past 2 weeks. Total BDI scores range from 0 to 63 , with higher scores reflecting greater lev- 
els of depressive symptoms.

To assess health related quality of life (QOL), the 12-item Short-Form health survey (SF-12), a short version of the 36-item SF health survey questionnaire, was used. ${ }^{14}$ Each of the 12 questions is rated on a 5-point Likert scale, and the sum of the scores can be calculated with a standardized scoring algorithm. Scores can be transformed into mental composite summary and physical composite summary scores. Higher scores on the SF-12 composite and total scores indicate higher QOL.

\section{Questionnaires on Mood and Sleep Disturbance}

To assess sleep quality and disturbances, the Pittsburgh Sleep Quality Index (PSQI), a self-reported questionnaire, was used. ${ }^{15}$ The PSQI yields a total score that ranges from 0 to 21 , with higher scores indicating more sleep difficulties. The PSQI has 7 subscales: subjective sleep quality, sleep latency, sleep duration, habitual sleep efficiency, sleep disturbances, use of sleeping medications, and daytime dysfunction. To evaluate insomnia status, participants were asked to rate the frequency of the following 4 insomnia symptoms in the prior month on a 4-point Likert scale $(1=$ never, $2=1-2$ times

Table 1. Baseline Characteristics in Subjects With Irritable Bowel Syndrome, Functional Dyspepsia, and Irritable Bowel Syndrom-Functional Dyspepsia Overlap

\begin{tabular}{|c|c|c|c|c|c|c|c|}
\hline & \multirow{2}{*}{$\begin{array}{c}\text { Normal } \\
(\mathrm{n}=1800)\end{array}$} & \multirow{2}{*}{$\begin{array}{l}\text { IBS Alone } \\
(\mathrm{n}=133)\end{array}$} & \multirow{2}{*}{$\begin{array}{l}\text { FD Alone } \\
(\mathrm{n}=62)\end{array}$} & \multirow{2}{*}{$\begin{array}{l}\text { IBS-FD } \\
\text { overlap } \\
(\mathrm{n}=36)\end{array}$} & \multicolumn{3}{|c|}{$P$-value } \\
\hline & & & & & $\begin{array}{l}\text { Normal vs } \\
\text { IBS Alone }\end{array}$ & $\begin{array}{l}\text { Normal vs } \\
\text { FD Alone }\end{array}$ & $\begin{array}{c}\text { Normal vs } \\
\text { IBS-FD }\end{array}$ \\
\hline Age & $58.6 \pm 6.7$ & $57.2 \pm 6.3$ & $58.2 \pm 6.6$ & $58.0 \pm 6.5$ & 0.017 & 0.639 & 0.581 \\
\hline Sex & & & & & 0.279 & 0.114 & 0.006 \\
\hline Female & $833(46.3 \%)$ & $68(51.1 \%)$ & $35(56.5 \%)$ & $25(69.4 \%)$ & & & \\
\hline Male & $967(53.7 \%)$ & $65(48.9 \%)$ & $27(43.5 \%)$ & $11(30.6 \%)$ & & & \\
\hline Alcohol & & & & & 0.370 & 0.605 & 0.316 \\
\hline Never & $898(49.9 \%)$ & $61(45.9 \%)$ & $33(53.2 \%)$ & $21(58.3 \%)$ & & & \\
\hline Current & $902(50.1 \%)$ & $72(54.1 \%)$ & $29(46.8 \%)$ & $15(41.7 \%)$ & & & \\
\hline Smoking & & & & & 0.154 & 0.599 & 0.664 \\
\hline Never & $1555(86.4 \%)$ & $109(82.0 \%)$ & $55(88.7 \%)$ & $32(88.9 \%)$ & & & \\
\hline Current & $245(13.6 \%)$ & $24(18.0 \%)$ & $7(11.3 \%)$ & $4(11.1 \%)$ & & & \\
\hline Education (yr) & & & & & 0.046 & 0.422 & 0.114 \\
\hline$<6$ & $200(11.1 \%)$ & $8(6.0 \%)$ & $7(11.3 \%)$ & $8(22.2 \%)$ & & & \\
\hline $6-12$ & $1183(65.7 \%)$ & $84(63.2 \%)$ & $45(72.6 \%)$ & $21(58.3 \%)$ & & & \\
\hline$>12$ & $417(23.2 \%)$ & $41(30.8 \%)$ & $10(16.1 \%)$ & $7(19.4 \%)$ & & & \\
\hline Income $\left(10^{4} \mathrm{won} / \mathrm{mo}\right)$ & & & & & 0.481 & 0.385 & 0.584 \\
\hline$<200$ & $422(23.4 \%)$ & $28(21.1 \%)$ & $17(27.4 \%)$ & $10(27.8 \%)$ & & & \\
\hline $200-400$ & $701(38.9 \%)$ & $48(36.1 \%)$ & $27(43.5 \%)$ & $11(30.6 \%)$ & & & \\
\hline$>400$ & $677(37.6 \%)$ & $57(42.9 \%)$ & $18(29.0 \%)$ & $15(41.7 \%)$ & & & \\
\hline Occupation & & & & & 0.978 & 0.349 & 0.136 \\
\hline White collar & $524(29.1 \%)$ & $38(28.6 \%)$ & $23(37.1 \%)$ & $16(44.5 \%)$ & & & \\
\hline Blue collar & $513(28.5 \%)$ & $39(29.3 \%)$ & $14(22.6 \%)$ & $8(22.2 \%)$ & & & \\
\hline Housekeeper & $763(42.4 \%)$ & $56(42.1 \%)$ & $25(40.3 \%)$ & $12(33.3 \%)$ & & & \\
\hline Marital status & & & & & 0.008 & 0.047 & 0.086 \\
\hline Married & $840(46.7 \%)$ & $78(58.6 \%)$ & $21(33.9 \%)$ & $22(61.1 \%)$ & & & \\
\hline Other & $960(53.3 \%)$ & $55(41.4 \%)$ & $41(66.1 \%)$ & $14(38.9 \%)$ & & & \\
\hline Fasting glucose (g/dL) & $97.1 \pm 20.8$ & $97.3 \pm 21.2$ & $99.5 \pm 25.3$ & $97.7 \pm 20.6$ & 0.919 & 0.388 & 0.865 \\
\hline $\mathrm{SBP}(\mathrm{mmHg})$ & $115.2 \pm 13.7$ & $113.5 \pm 13.6$ & $113.1 \pm 12.1$ & $110.5 \pm 11.8$ & 0.178 & 0.232 & 0.043 \\
\hline $\mathrm{DBP}(\mathrm{mmHg})$ & $75.4 \pm 9.6$ & $74.8 \pm 9.4$ & $74.3 \pm 8.7$ & $72.9 \pm 9.2$ & 0.479 & 0.370 & 0.113 \\
\hline Triglycerides (mg/dL) & $142.8 \pm 98.4$ & $147.3 \pm 97.7$ & $156.7 \pm 90.4$ & $143.5 \pm 77.3$ & 0.613 & 0.272 & 0.963 \\
\hline $\mathrm{HDL}(\mathrm{mg} / \mathrm{dL})$ & $49.4 \pm 12.7$ & $51.9 \pm 13.4$ & $48.6 \pm 11.7$ & $54.6 \pm 14.1$ & 0.025 & 0.624 & 0.014 \\
\hline
\end{tabular}

IBS, irritable bowel syndrome; FD, functional dyspepsia; IBS-FD overlap, defined as the fulfillment of the criteria of both IBS and FD; SBP, systolic blood pressure; DBP, diastolic blood pressure; HDL, high-density lipoprotein. 
per week, $3=3-4$ times per week, and $4=$ more than 5 times per week): difficulty initiating sleep, difficulty maintaining sleep, experience of early morning awakening, and low mood in the morning. Individuals who indicated that they experienced at least 1 of these 4 symptoms more than 3-4 times per week (score $\geq 3$ on any 1 item) were categorized as having insomnia symptoms, regardless of their use of sleep medicine. ${ }^{16}$ The Epworth Sleepiness Scale (ESS), is an 8-item questionnaire for daytime sleepiness with scores ranging from 0 to 24, with higher scores indicating greater sleepiness. The ESS was also used to assess the general level of daytime sleepiness. ${ }^{17}$

\section{Statistical Methods}

Data are expressed as means \pm standard deviations for continuous variables or numbers and as percentages for categorical variables. The analyses of continuous and categorical variables to assess differences among the 4 groups in this study were performed

A
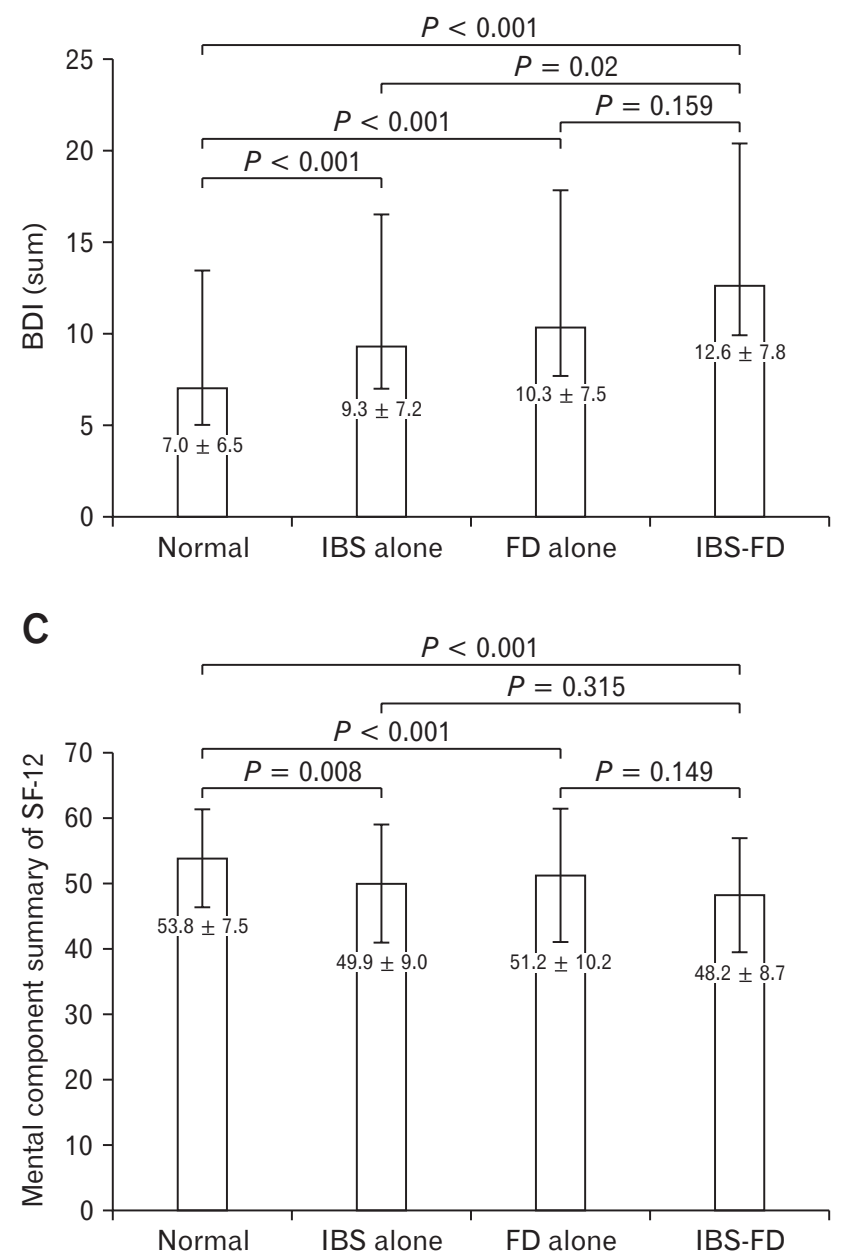

by one-way ANOVA, using the Scheffe post-hoc test or the $\chi^{2}$ test for comparison. Logistic regression analysis was performed to estimate the odds ratio (OR) with $95 \%$ confidence interval (CI) for the correlation between IBS and FD. Covariates included in the multivariate models were age, sex, income, occupation, educational level, smoking status, BMI, BDI score, alcohol consumption, and physical activity (metabolic equivalents per hour daily). To explore the mediators of the correlation between IBS and FD, subjects with FD were subcategorized into 4 groups: without both FD and IBS, with FD and without IBS, without FD and with IBS, and with both FD and IBS. $P<0.05$ was considered statistically significant. All statistical analyses were performed using SAS statistical software (SAS 9.4, SAS Institute, Cary, NC, USA).

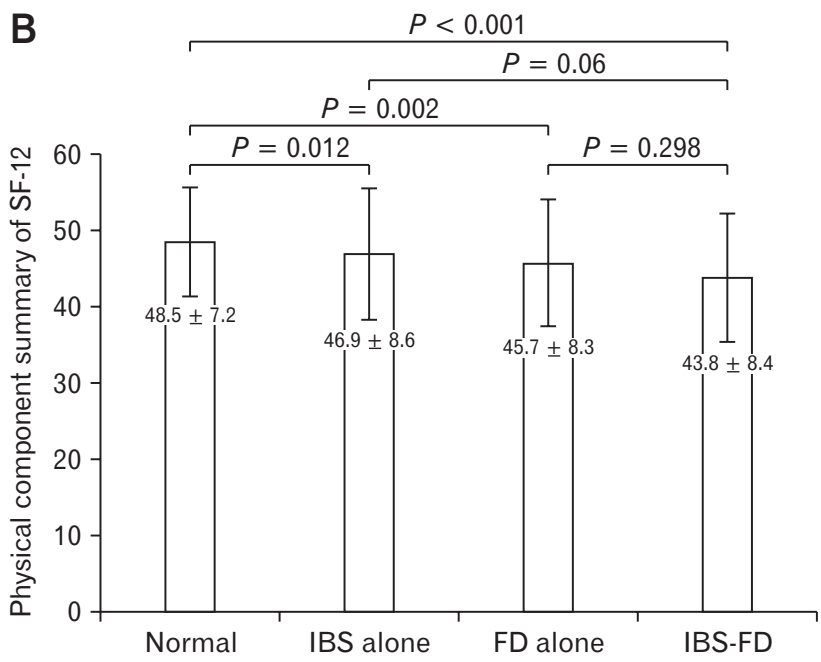

Figure 1. Depression and quality of life in functional gastrointestinal disorders. The (A) Beck Depression Inventory (BDI) scores, (B) physical component summary of the 12-item Short-Form health survey (SF-12), and (c) mental component summary of the SF-12, for subjects with irritable bowel syndrome (IBS) alone, functional dyspepsia (FD) alone, and IBS-FD overlap, as compared with normal healthy controls. IBS-FD overlap, defined as the fulfillment of the criteria of both IBS and FD. 


\section{Results}

\section{Baseline Characteristics}

Among 2031 eligible subjects, 169 (8.3\%) subjects were diagnosed with IBS, 98 (4.8\%) with FD, and 36 (1.8\%) with IBS/FD overlap. The age- and sex-adjusted prevalence rates of IBS, FD, and IBS-FD overlap were $8.01 \%$ (95\% CI, 6.8-9.2\%), 4.8\% (95\% CI, 3.9-5.8\%), and $1.8 \%$ (95\% CI, 1.2-2.4\%), respectively.

Table 1 shows the characteristics of the study subjects according to FD or IBS status. The mean age of patients with IBS alone was less than that of subjects without IBS or dyspepsia, and IBS-FD overlap was more prevalent in females than in males. IBS was more prevalent in subjects with a higher educational level, and there were differences in marital status between IBS and FD.

A

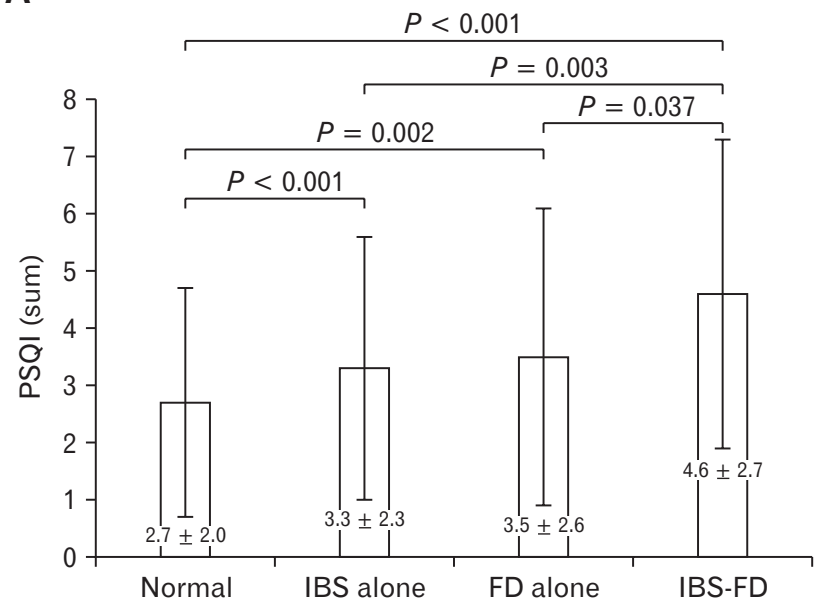

C

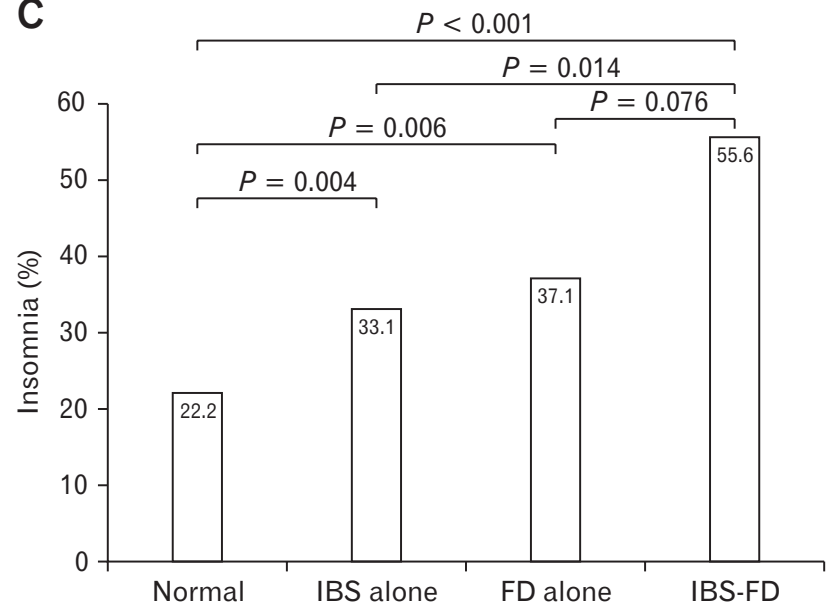

\section{Depression and Quality of Life}

Figure 1 shows the depression and QOL status according to the functional bowel disorder. The BDI, which indicates the degree of depression, was higher in patients with IBS, FD, and IBS-FD overlap than in healthy subjects. The BDI of subjects with IBS-FD overlap was highest, and there were significant differences in the BDI between IBS or FD alone and IBS-FD overlap groups (Fig. 1A). Both the physical and mental components of the SF-12, representing QOL, were significantly lower in IBS alone, FD alone, or IBS-FD overlap, compared to healthy subjects (Fig. 1B and 1C).

\section{Sleep Impairment in Irritable Bowel Syndrome or Functional Dyspepsia}

Figure 2 shows that sleep impairment was more prominent in subjects with IBS alone, FD alone, or IBS-FD overlap, compared

B

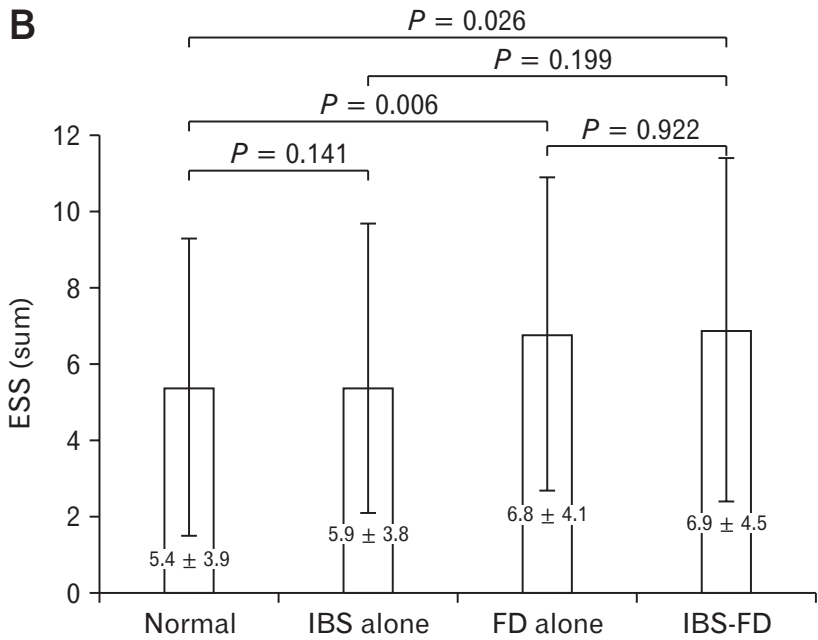

Figure 2. Sleep impairment in functional gastrointestinal disorders. (A) The Pittsburgh Sleep Quality Index (PSQI), (B) Epworth Sleepiness Scale (ESS) scores, and (C) insomnia in subjects with irritable bowel syndrome (IBS) alone, functional dyspepsia (FD) alone, and IBS-FD overlap, as compared with normal healthy controls. IBS-FD, IBS-FD overlap, defined as the fulfillment of the criteria of both IBS and FD. 
Table 2. Association of Pittsburgh Sleep Quality Index, Epworth Sleepiness Scale, and Insomnia With Irritable Bowel Syndrome and Functional Dyspepsia

\begin{tabular}{lcc}
\hline & $\begin{array}{c}\text { Crude } \\
\text { OR }(95 \% \mathrm{CI}) P \text {-value }\end{array}$ & $\begin{array}{c}\text { Multivariate } \\
\text { OR }(95 \% \mathrm{CI}) P \text {-value }\end{array}$ \\
\hline PSQI & reference & reference \\
Normal & $1.57(0.93-2.66) 0.092$ & $1.31(0.75-2.26) 0.340$ \\
IBS alone & $3.38(1.87-6.13)<0.001$ & $2.68(1.43-5.01) 0.002$ \\
FD alone & & \\
IBS-FD overlap & $5.24(2.59-10.59)<0.001$ & $3.88(1.83-8.19)<0.001$ \\
ESS & reference & reference \\
Normal & $1.56(0.91-2.67) 0.106$ & $1.36(0.79-2.36) 0.268$ \\
IBS alone & $2.55(1.33-4.89) 0.05$ & $2.21(1.14-4.30) 0.019$ \\
FD alone & & $2.47(1.08-5.67) 0.032$ \\
IBS-FD overlap & $2.92(1.30-6.55) 0.092$ & reference \\
Insomnia & reference & $1.44(0.95-2.17) 0.083$ \\
Normal & $1.76(1.20-2.58) 0.003$ & $1.50(0.85-2.66) 0.163$ \\
IBS alone & $2.01(1.18-3.42) 0.010$ & $0.84(1.39-5.82) 0.004$ \\
FD alone & $4.08(2.08-8.00)<0.001$ & 2.84 \\
IBS-FD overlap &
\end{tabular}

PSQI, Pittsburgh sleep quality index (PSQI); ESS, Epworth sleepiness scale; IBS, irritable bowel syndrome; FD, functional dyspepsia; IBS-FD overlap, defined as the fulfillment of the criteria of both IBS and FD.

Odds ratio was calculated for the comparison of non-IBS patients with nonFD patients.

Crude data are adjusted for age and sex; the multivariate data are adjusted for age and sex plus income, occupation, educational level, smoking status, body mass index, Beck depression inventory score, alcohol consumption, and physical activity (metabolic equivalents per hour daily).

to healthy subjects. The global PSQI score in subjects with functional bowel disorders was significantly higher than that of healthy subjects (Fig. 2A). In addition, the proportion of subjects with PSQI total scores higher than 6 was greater in IBS alone $(13.5 \%$, $P<0.01)$, FD alone $(25.8 \%, P<0.01)$, and IBS-FD overlap (36.0\%, $P<0.01)$, compared to healthy subjects $(9.1 \%)$. The ESS scores, which reflect the extent of daytime sleepiness, were significantly higher in subjects with FD alone and IBS-FD overlap, compared to healthy subjects (Fig. 2B). Moreover, insomnia occurred more frequently in IBS alone, FD alone, and IBS-FD overlap, compared to healthy subjects (Fig. 2C). However, the mean hours of sleep duration were not different between groups.

Table 2 shows the multivariate analysis of the correlation between functional bowel disorders and sleep disturbance. After all other factors (age, sex, income, occupation, educational level, smoking status, alcohol consumption, BMI, BDI score, and physical activity) were adjusted, FD was found to have a significant effect on sleep quality (OR, 2.68; CI, 1.43-5.01) and daytime sleepiness (OR, 2.21; CI, 1.14-4.30), whereas IBS alone was not indepen- dently associated with sleep quality, insomnia, or daytime sleepiness. In addition, subjects who reported concurrent FD and IBS were more likely to have poorer sleep quality (OR, 3.88; CI, 1.83-8.19), insomnia (OR, 2.84; CI, 1.39-5.82), and daytime sleepiness (OR, 2.47; CI, 1.08-5.67).

\section{Discussion}

In this population-based study using the Rome III criteria, the prevalence of IBS, FD, and IBS-FD overlap was $8.0 \%, 4.8 \%$, and $1.8 \%$, respectively. In a previous population-based Korean study that used the Rome II criteria, the corresponding prevalence rates were $9.6 \%, 9.5 \%$, and $1.3 \%{ }^{18}$ Moreover, we found a significant correlation between functional bowel disorders and self-reported sleep disturbances; in particular, the highest correlation with poor sleep quality was found in subjects with IBS-FD overlap.

The association between sleep disturbance and organic gastrointestinal diseases is well established. Previous studies showed that sleep disorders were more common in a gastroesophageal reflux disease (GERD) cohort than in a comparison cohort (hazard ratio = 2.69; CI, 1.83-3.94), ${ }^{19}$ and that individuals with peptic ulcers showed more daytime sleepiness compared to patients with other medical conditions, such as gynecological disorders, neurological disorders, or heart disease..$^{20}$ In the absence of organic disease, the presence of gastrointestinal symptoms exhibited a relationship with sleep disturbance, and multiple gastrointestinal symptoms tended to have a stronger association than single symptoms. ${ }^{5,21}$ Therefore, it could be considered natural that FGIDs, which manifest various chronic gastrointestinal symptoms including abdominal pain, are accompanied by sleep disturbance. In accordance with our findings, the prevalence of sleep disturbances in patients with IBS or FD was higher than that in healthy subjects in previous studies. ${ }^{7,22}$

Sleep impairment and digestive symptoms are closely related. Sleep disturbance in patients with FGID could be due to existing, chronic painful physical stimuli, including gastrointestinal symptoms that cause difficulty in sleeping, inability to resume sleep once awake, and a shorter sleep duration. ${ }^{23}$ In addition, sleep deprivation itself has been shown to be an aggravating factor of unpleasant nextday abdominal symptoms in women with IBS. ${ }^{24}$

Other explanations for the high prevalence of sleep disturbances in patients with FGIDs could be related to psychological distress, such as depression or anxiety, which are commonly reported in these patients. ${ }^{6}$ In a study in Singapore, $51 \%$ of refractory FGID patients at a tertiary gastroenterology unit had significant anxiety or depression compared with controls. ${ }^{25}$ Acute or chronic psychosocial stress- 
ors frequently precede the onset and exacerbations of FGID. ${ }^{26,27}$ Approximately two-thirds of patients with IBS who are referred to secondary care centers have some form of psychological distress, commonly anxiety or depression. ${ }^{28}$ Notably, in another study, Robert et $\mathrm{al}^{29}$ showed that patients with both IBS and depression complained of more sleep issues than both patients with IBS without depression and healthy subjects. Our study also confirmed that depression was more prevalent in patients with IBS or FD than in healthy subjects. Moreover, in patients with FD, the self-reporting of poor sleep was found to be positively correlated with gastrointestinal symptom severity, and poor sleep was strongly associated with anxiety and depression. ${ }^{30}$ Several studies have shown that psychological distress may alter gut immunity and permeability, which is being increasingly recognized as a pathophysiological mechanism in FGID. ${ }^{31,32}$

Taking a different approach, several studies that used polysomnography in IBS showed that the proportion of rapid eye movement sleep was markedly increased, ${ }^{33,34}$ with more frequent sleep fragmentation and awakening in IBS patients. ${ }^{35}$ In another study, rapid eye movement sleep, arousals, and waking was associated with increased colon motility.

Our study revealed that the global PSQI scores, ESS scores, and prevalence of insomnia were higher in subjects with IBS or FD alone than in healthy subjects. Interestingly, the likelihood of sleep problems was greater in FD patients than in IBS patients. Due to limited data pertaining to the increased prevalence of sleep impairment in FD than in IBS, further studies are needed.

Our study also examined QOL using the SF-12 questionnaire. The scores for both the physical and mental components were lower in patients with IBS alone, FD alone, or IBS-FD overlap, compared to healthy controls. Patients with IBS experience significant impairment in health-related QOL. ${ }^{37}$ Several studies also reported that QOL in FD was reduced as compared with that of healthy subjects. Moreover, QOL showed a significant association with global PSQI scores in patients with FD.,

In our study, the IBS-FD overlap group showed the greatest likelihood of sleep disturbance in all scoring parameters when compared to healthy controls. The BDI score was also higher in subjects with IBS-FD overlap than in subjects with FD or IBS alone. The degree of overlap between IBS and FD varied from 15\% to 42\%, depending on the diagnostic criteria that was used. ${ }^{38}$ Abnormalities of the psychosocial or central nervous systems that affect gastrointestinal motility, as well as altered immune responses, could be considered as possible risk factors for IBS-FD overlap. ${ }^{39}$ Depressed mood was reported to have a significant association with $\mathrm{FD}$, and there was a greater frequency of decreased QOL and depressed mood in patients with IBS-FD overlap than in patients with IBS or FD alone. ${ }^{40}$ Park et $\mathrm{al}^{41}$ reported that the somatization and anxiety scores were higher in IBS-FD overlap than in either IBS or FD alone. Along with an increase in the number of co-existing FGIDs, there was a corresponding increase in the prevalence of anxiety and depression. ${ }^{42}$

This study has several limitations. Although this was a population-based cohort study, the enrolled subjects were all middle-aged. Therefore, the prevalence of IBS or FD may not be generalizable to the general population. Moreover, there were some differences between healthy subjects and patients with FGIDs in sex, education level, and marital status, which might affect the prevalence of sleep disturbance. However, we calculated odds ratio after adjusting for many variables, including age, sex, education level, etc. In addition, since the prevalence of sleep issues such as insomnia tends to be higher among older women and men, sleep issues might have occurred more frequently in our study. Finally, although there was an obvious correlation between FGID and sleep disturbances, we were unable to determine causality owing to the cross-sectional design and complex pathophysiology of FGID.

In conclusion, this study provides evidence for the association between sleep disturbances and FGIDs in a Korean community; this association was much stronger in the subjects with concurrent FD and IBS. In particular, sleep quality was more significantly impaired when both FD and IBS were present. Our results demonstrate that QOL and psychological stresses are significantly correlated with FD and IBS, and that the correlation is much stronger in patients with IBS-FD overlap. Further studies with a larger sample size and a prospective approach are warranted to assess a possible causal relationship between sleep and FGIDs.

Financial support: This research was supported by the Korea Centers for Disease Control and Prevention (2012-E71005-00, 2013-E71005-00) and by the Bio and Medical Technology Development Program of the NRF funded by the Korean government, MSIP (NRF-2015M3A9B6027142). This study was partly supported by a Korea University Grant.

\section{Conflicts of interest: None.}

Author contributions: Rok Seon Choung and Chol Shin developed the original concept, designed the study, and advised in the writing of the manuscript; Seung Young Kim wrote the manuscript; Seung Ku Lee analyzed the data; Jung Wan Choe, Sung Woo 
Jung, and Ja Seol Koo reviewed the manuscript and advised in the writing of the manuscript; and Jong Jin Hyun and Sang Woo Lee reviewed the manuscript, and assisted with English-language issues and the revision of the manuscript.

\section{References}

1. Weissman MM, Greenwald S, Niño-Murcia G, Dement WC. The morbidity of insomnia uncomplicated by psychiatric disorders. Gen Hosp Psychiatry 1997;19:245-250.

2. Parish JM. Sleep-related problems in common medical conditions. Chest 2009;135:563-572.

3. Smagula SF, Koh WP, Wang R, Yuan JM. Chronic disease and lifestyle factors associated with change in sleep duration among older adults in the Singapore Chinese health study. J Sleep Res 2016;25:57-61.

4. Dickman R, Green C, Fass SS, et al. Relationships between sleep quality and $\mathrm{pH}$ monitoring findings in persons with gastroesophageal reflux disease. J Clin Sleep Med 2007;3:505-513.

5. Yamawaki H, Futagami S, Shimpuku M, et al. Impact of sleep disorders, quality of life and gastric emptying in distinct subtypes of functional dyspepsia in Japan. J Neurogastroenterol Motil 2014;20:104-112.

6. Morito Y, Aimi M, Ishimura N, et al. Association between sleep disturbances and abdominal symptoms. Intern Med 2014;53:2179-2183.

7. Lacy BE, Everhart K, Crowell MD. Functional dyspepsia is associated with sleep disorders. Clin Gastroenterol Hepatol 2011;9:410-414.

8. Ford AC, Bercik P, Morgan DG, Bolino C, Pintos-Sanchez MI, Moayyedi P. Characteristics of functional bowel disorder patients: a crosssectional survey using the Rome III criteria. Aliment Pharmacol Ther 2014;39:312-321.

9. Kaji M, Fujiwara Y, Shiba M, et al. Prevalence of overlaps between GERD, FD and IBS and impact on health-related quality of life. J Gastroenterol Hepatol 2010;25:1151-1156.

10. Baik I, Shin C. Prospective study of alcohol consumption and metabolic syndrome. Am J Clin Nutr 2008;87:1455-1463.

11. Tack J, Talley NJ, Camilleri M, et al. Functional gastroduodenal disorders. Gastroenterology 2006;130:1466-1479.

12. Longstreth GF, Thompson WG, Chey WD, Houghton LA, Mearin F, Spiller RC. Functional bowel disorders. Gastroenterology 2006;130:1480-1491.

13. Beck AT, Ward CH, Mendelson M, Mock J, Erbaugh J. An inventory for measuring depression. Arch Gen Psychiatry 1961;4:561-571.

14. Ware JE, Kosinsk M, Keller SD. A 12-Item short form health survey: construction of scales and preliminary tests of reliability and validity. Med Care 1996;34:220-233.

15. Buysse DJ, Reynolds CF 3rd, Monk TH, Berman SR, Kupfer DJ. The pittsburgh sleep quality index: a new instrument for psychiatric practice and research. Psychiatry Res 1989;28:193-213.

16. Suh S, Yang HC, Fairholme CP, Kim H, Manber R, Shin C. Who is at risk for having persistent insomnia symptoms? A longitudinal study in the general population in Korea. Sleep Med 2014;15:180-186.

17. Johns MW. A new method for measuring daytime sleepiness: the ep- worth sleepiness scale. Sleep 1991;14:540-545.

18. Lee SY, Lee KJ, Kim SJ, Cho SW. Prevalence and risk factors for overlaps between gastroesophageal reflux disease, dyspepsia, and irritable bowel syndrome: a population-based study. Digestion 2009;79:196-201.

19. You ZH, Perng CL, Hu LY, et al. Risk of psychiatric disorders following gastroesophageal reflux disease: a nationwide population-based cohort study. Eur J Intern Med 2015;26:534-539.

20. Stroe AF, Roth T, Jefferson C, et al. Comparative levels of excessive daytime sleepiness in common medical disorders. Sleep Med 2010;11:890896.

21. Gathaiya N, Locke GR 3rd, Camilleri M, Schleck CD, Zinsmeister AR, Talley NJ. Novel associations with dyspepsia: a community-based study of familial aggregation, sleep dysfunction and somatization. Neurogastroenterol Motil 2009;21:922-e69.

22. Miwa H. Life style in persons with functional gastrointestinal disorders-large-scale internet survey of lifestyle in Japan. Neurogastroenterol Motil 2012;24:464-471, e217.

23. Ohayon MM. Relationship between chronic painful physical condition and insomnia. J Psychiatr Res 2005;39:151-159.

24. Jarrett M, Heitkemper M, Cain KC, Burr RL, Hertig V. Sleep disturbance influences gastrointestinal symptoms in women with irritable bowel syndrome. Dig Dis Sci 2000;45:952-959.

25. Wang YT, Kwok KF, Tan SM, et al. Comprehensive psychological intervention to improve outcome in functional gastrointestinal disorder: a cohort study. Singapore Med J 2015;56:385-392.

26. Van Oudenhove L, Demyttenaere K, Tack J, Aziz Q. Central nervous system involvement in functional gastrointestinal disorders. Best Pract Res Clin Gastroenterol 2004;18:663-680.

27. Budavari AI, Olden KW. Psychosocial aspects of functional gastrointestinal disorders. Gastroenterology Clin North Am 2003;32:477-506.

28. Spiller R, Aziz Q, Creed F, et al. Guidelines on the irritable bowel syndrome: mechanisms and practical management. Gut 2007;56:17701798.

29. Robert JJ, Orr WC, Elsenbruch S. Modulation of sleep quality and autonomic functioning by symptoms of depression in women with irritable bowel syndrome. Dig Dis Sci 2004;49:1250-1258.

30. Fass R, Fullerton S, Tung S, Mayer EA. Sleep disturbances in clinic patients with functional bowel disorders. Am J Gastroenterol 2000;95:11952000.

31. Wouters MM, Van Wanrooy S, Nguyen A, et al. Psychological comorbidity increases the risk for postinfectious IBS partly by enhanced susceptibility to develop infectious gastroenteritis. Gut 2016;65:1279-1288.

32. Vanuytsel T, van Wanrooy S, Vanheel H, et al. Psychological stress and corticotropin-releasing hormone increase intestinal permeability in humans by a mast cell-dependent mechanism. Gut 2014;63:1293-1299.

33. Kumar D, Thompson PD, Wingate DL, Vesselinova-Jenkins CK, Libby G. Abnormal REM sleep in the irritable bowel syndrome. Gastroenterology 1992;103:12-17.

34. Orr WC, Crowell MD, Lin B, Harnish MJ, Chen JD. Sleep and gastric function in irritable bowel syndrome: derailing the brain-gut axis. Gut 1997;41:390-393.

35. Rotem AY, Sperber AD, Krugliak P, Freidman B, Tal A, Tarasiuk A. 
Polysomnographic and actigraphic evidence of sleep fragmentation in patients with irritable bowel syndrome. Sleep 2003;26:747-752.

36. Furukawa Y, Cook IJ, Panagopoulos V, McEvoy RD, Sharp DJ, Simula M. Relationship between sleep patterns and human colonic motor patterns. Gastroenterology 1994;107:1372-1381.

37. Gralnek IM, Hays RD, Kilbourne A, Naliboff B, Mayer EA. The impact of irritable bowel syndrome on health-related quality of life. Gastroenterology 2000;119:654-660.

38. Ford AC, Marwaha A, Lim A, Moayyedi P. Systematic review and meta-analysis of the prevalence of irritable bowel syndrome in individuals with dyspepsia. Clin Gastroenterol Hepatol 2010;8:401-409.

39. Suzuki H, Hibi T. Overlap syndrome of functional dyspepsia and irri- table bowel syndrome - are both diseases mutually exclusive? J Neurogastroenterol Motil 2011;17:360-365.

40. Lee HJ, Lee SY, Kim JH, et al. Depressive mood and quality of life in functional gastrointestinal disorders: differences between functional dyspepsia, irritable bowel syndrome and overlap syndrome. Gen Hosp Psychiatry 2010;32:499-502.

41. Park JM, Choi MG, Cho YK, et al. Functional gastrointestinal disorders diagnosed by Rome III questionnaire in Korea. J Neurogastroenterol Motil 2011;17:279-286.

42. Pinto-Sanchez MI, Ford AC, Avila CA, et al. Anxiety and depression increase in a stepwise manner in parallel with multiple FGIDs and symptom severity and frequency. Am J Gastroenterol 2015;110:1038-1048. 